\title{
An integral equation study of a simple point charge model of water
}

\author{
M. Lombardero, C. Martín, and S. Jorge \\ Instituto de Química Física Rocasolano, CSIC, Serrano 119, E-28006 Madrid, Spain \\ and Departamento de Química Física I, Universidad Complutense, E-28040 Madrid, Spain \\ F. Lado \\ Department of Physics, North Carolina State University, Raleigh, North Carolina 27695-8202 \\ E. Lomba ${ }^{\text {a) }}$ \\ Instituto de Química Física Rocasolano, CSIC, Serrano 119, E-28006 Madrid, Spain
}

(Received 11 June 1998; accepted 7 October 1998)

\begin{abstract}
We present an extensive integral equation study of a simple point charge model of water for a variety of thermodynamic states ranging from the vapor phase to the undercooled liquid. The calculations are carried out in the molecular reference-hypernetted chain approximation and the results are compared with extensive molecular dynamics simulations. Use of a hard sphere fluid as a reference system to provide the input reference bridge function leads to relatively good thermodynamics. However, at low temperatures the computed microscopic structure shows deficiencies that probably stem from the lack of orientational dependence in this bridge function. This is in marked contrast with results previously obtained for systems that, although similarly composed of angular triatomic molecules, do not tend to the tetrahedral coordinations that are characteristic of water. (C) 1999 American Institute of Physics. [S0021-9606(99)52202-2]
\end{abstract}

\section{INTRODUCTION}

The description of liquid water from a microscopic perspective hinges on the knowledge of its interaction potential, which in particular should be able to account for the tetrahedral coordination of the solid and the larger coordination numbers - and consequently larger density - found upon melting. On the one hand, several attempts have been made to attain a pure $a b$ initio description of liquid water, ${ }^{1}$ but the practical limitations to small sample sizes and short time spans considerably constrain the range of problems to which this sort of approach can be applied. On the other hand, a large and growing number of simple effective intermolecular potentials has been proposed. Without attempting to be exhaustive, we can catalog those that deal with the molecule as a rigid entity (TIP4, ${ }^{2} \mathrm{SPC},{ }^{3} \mathrm{SPCE}^{4}$ ), those that allow for flexible bonds, ${ }^{5}$ those that explicitly incorporate $\mathrm{N}$-body effects through polarizability, ${ }^{6}$ and finally those that allow for dissociation, such as the central force model. ${ }^{7}$

With such varied competition for the title of "the" model of water, it is obviously difficult to venture a sound (and lasting) assessment of which of these models might be considered the most adequate. Nevertheless, one of the most successful simple models, shown able to reproduce the most significant features of liquid water, has turned out to be the simple point charge (SPC) model ${ }^{3}$ or its extended version ${ }^{4}$ (SPCE). A large number of works have concentrated on computer simulation studies of this model to examine not only structure, thermodynamics, and dielectric properties, ${ }^{8}$ but also phase equilibrium. ${ }^{9}$ In contrast, theoretical approaches have been constrained by the formidable task of appropriately dealing with the threefold orientational degrees

${ }^{a)}$ Electronic mail: E.Lomba@iqfr.csic.es of freedom of the molecules and hence most theoretical efforts have gone into RISM-like (reference interactive site model) theories, either in its original form ${ }^{10}$ or in the proper RISM equation of Chandler, Silbey, and Ladanyi, ${ }^{11}$ applied to water by Lue and Blankschtein. ${ }^{12}$

Very recently, the present authors have cast the molecular Ornstein-Zernike (OZ) equation into a form suitable for application to real molecular fluids composed of nonlinear molecules. ${ }^{13,14}$ Calculations performed on realistic models for $\mathrm{H}_{2} \mathrm{~S}$ and $\mathrm{SO}_{2}$ showed that the molecular $\mathrm{OZ}$ equation with a reference-hypernetted chain (RHNC) closure employing a simple hard-sphere reference bridge function was very accurate in its predictions of the microscopic structure, thermodynamics, and dielectric constant (when the proper charge distribution was used), even for a system with a relatively large degree of anisotropy and polarity such as $\mathrm{SO}_{2}$.

This success suggested that the simple RHNC recipe might be put to a more stringent test, namely the study of water. This is the task we undertake in the present work. Since the SPCE model for water is very similar to the pointcharge model successfully used in Ref. 14 for $\mathrm{H}_{2} \mathrm{~S}$, one might expect that the same procedure, using a spherically symmetric reference bridge function, should perform nicely for water as well. We shall see, however, that crucial differences arise in the case of water that are closely associated with the tendency of this substance to form tetrahedral coordinations, a tendency lacking in liquid $\mathrm{H}_{2} \mathrm{~S}$ or $\mathrm{SO}_{2}$. We conclude that one needs an orientationally dependent reference bridge function that reflects this feature to construct a reliable RHNC recipe for liquid water.

In Sec. II, we briefly sketch the essential aspects of the theory, with some emphasis on the optimization procedures 
utilized throughout these calculations. In Sec. III we present a detailed comparison of the RHNC results with data from molecular dynamics simulations, ${ }^{15}$ paying particular attention to properties at room temperature and on the isochore $\rho=1 \mathrm{~g} / \mathrm{cm}^{3}$. Here the tetrahedral ordering of water is made evident by appealing to an analysis of the three-body correlation function. In the low-temperature, high-density regime the microscopic structure obtained from the theory is far from satisfactory. The structural data are more accurate at somewhat higher temperatures and especially so in the supercritical regime. Surprisingly, the simple RHNC theory performs reasonably well for the thermodynamics over the whole temperature range studied.

\section{THEORY}

Since the details of the numerical procedure can be found in Ref. 14, we will simply recall here the essential equations that constitute the RHNC integral equation approximation. Once again our starting point is the $\mathrm{OZ}$ relation, which for molecular fluids reads

$$
\gamma(12)=\frac{\rho}{8 \pi^{2}} \int d \mathbf{3} c(13)[c(32)+\gamma(32)],
$$

where $\rho$ is the number density and $\int d \mathbf{3}=\int d \boldsymbol{r}_{3} d \omega_{3}$ denotes integration over positions and orientations of molecule 3 . Here $c(12)$ is the direct correlation function and $\gamma(12)$ $=g(12)-1-c(12)$ is the indirect correlation function, where $g(12)=g\left(\mathbf{r}_{12}, \omega_{1}, \omega_{2}\right)$ is the molecular pair distribution function.

The OZ relation is supplemented with a closure that establishes a functional relation between $c(12)$ and $\gamma(12)$. In the case of the RHNC approximation, this is

$$
c(12)=\exp \left[-\beta u(12)+\gamma(12)+B_{0}(12)\right]-\gamma(12)-1,
$$

where $\beta=1 / k_{B} T, u(12)$ is the intermolecular pair potential, and $B_{0}(12)$ the so-called bridge function, which is obtained from a reference system whose physical parameters may be optimized by some criterion. Following Ref. 14, we use here as a reference system the hard sphere fluid. The only optimization parameter available is then the hard sphere diameter $d$, which we adjust to satisfy the criterion of free energy minimization. ${ }^{16}$ This leads to the condition

$$
\rho \int d \boldsymbol{r}\left[g_{000}^{00}(r)-g_{\mathrm{HS}}(r ; d)\right] \frac{\partial B_{\mathrm{HS}}(r ; d)}{\partial d}=0 .
$$

The hard sphere (HS) fluid bridge function $B_{\mathrm{HS}}(r ; d)$ is obtained from the Verlet-Weis-Henderson-Grundke ${ }^{17,18}$ parametrization. The solution of Eqs. (1)-(2) proceeds through expansions in angular basis functions:

$$
\begin{aligned}
X(12)= & \sum_{l_{1}, l_{2}, m, n_{1}, n_{2}}\left[\left(2 l_{1}+1\right)\left(2 l_{2}+1\right)\right]^{1 / 2} \\
& \times X_{l_{1} l_{2} m}^{n_{1} n_{2}}\left(r_{12}\right) D_{m n_{1}}^{l_{1}}\left(\omega_{1}\right) * D_{m n_{2}}^{l_{2}}\left(\omega_{2}\right)^{*},
\end{aligned}
$$

where $\omega=(\phi, \theta, \chi)$ are the Euler angles referred to the axial line $\boldsymbol{r}_{12}$ joining the molecular centers and $D_{m n}^{l}(\omega)$ are generalized spherical harmonic coefficients in the convention of

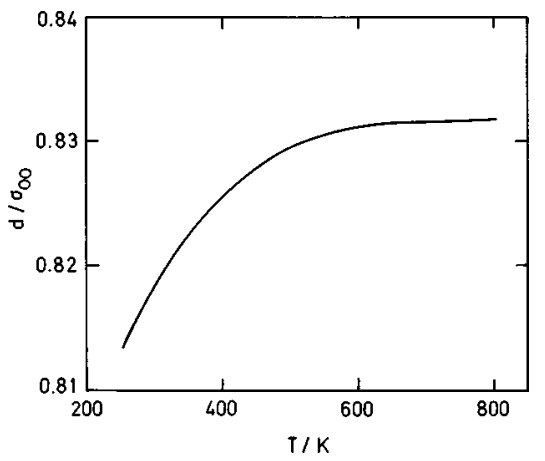

FIG. 1. Temperature dependence of the optimized reference hard sphere diameter $d$ on the isochore $\rho=0.997 \mathrm{~g} / \mathrm{cm}^{3}$.

Rose. ${ }^{19}$ Thus one sees that $g_{000}^{00}(r)$ in Eq. (3) is the first term in the angular expansion of $g(12)$ and is just the center-tocenter (or radial) distribution function. Specific expressions to evaluate thermodynamic and dielectric properties as well as to calculate site-site correlation functions from the expansion coefficients can be found in Refs. 13 and 14. An alternative procedure for the evaluation of site-site functions from the molecular $g(12)$ has recently been described by Richardi et al. $^{20}$

Once the interaction model has been chosen, one can iteratively solve the nonlinear system of Eqs. (1)-(2) until convergence is reached for a given reference diameter $d$, systematically modify $d$, and repeat the calculation until condition (3) is further satisfied. This straightforward implementation of the optimization is however somewhat tedious, since it requires repeated solutions of the fairly intricate integral equation. Thus, we resort to the strategy devised by Lombardero et al. ${ }^{21}$ for systems of linear molecules. In this scheme, the hard sphere diameter of the reference system which will be correlated only with the single set of LennardJones parameters $\epsilon, \sigma$ that occurs in the SPC and SPCE models for the $\mathrm{O}-\mathrm{O}$ interaction - is determined as a function of density and temperature according to the prescription

$$
d=\alpha\left[25-\ln \left(k_{B} T / \epsilon\right)\right]\left[25+\ln \left(\rho \sigma^{3}\right)\right] .
$$

The length parameter $\alpha$ in turn is determined using as a standard the optimized diameter $d$ calculated by means of Eq. (3) at a high density state $\left(\rho=0.997 \mathrm{~g} / \mathrm{cm}^{3}\right)$ for each isotherm. Obviously, for extensive computations, this procedure will produce a considerable saving of computer time. The temperature evolution of the optimized diameter for the high density state is displayed in Fig. 1.

We now recall the definition of the SPCE model for water as proposed by Berendsen et al. ${ }^{4}$ These authors included a self-energy correction in the original SPC model; the new interaction is characterized by three point masses with $\mathrm{OH}$ distances of $1 \AA$ and angle $\widehat{\mathrm{HOH}}=109.47^{\circ}$ (i.e., the tetrahedral angle). Point charges equal to $-0.8476 e$ and $+0.4238 e$ are located at the $\mathrm{O}$ and $\mathrm{H}$ sites, respectively, where $e$ is the elementary charge. In addition to the Coulomb interactions, the model incorporates dispersion forces via a single Lennard-Jones interaction between the oxygens: 


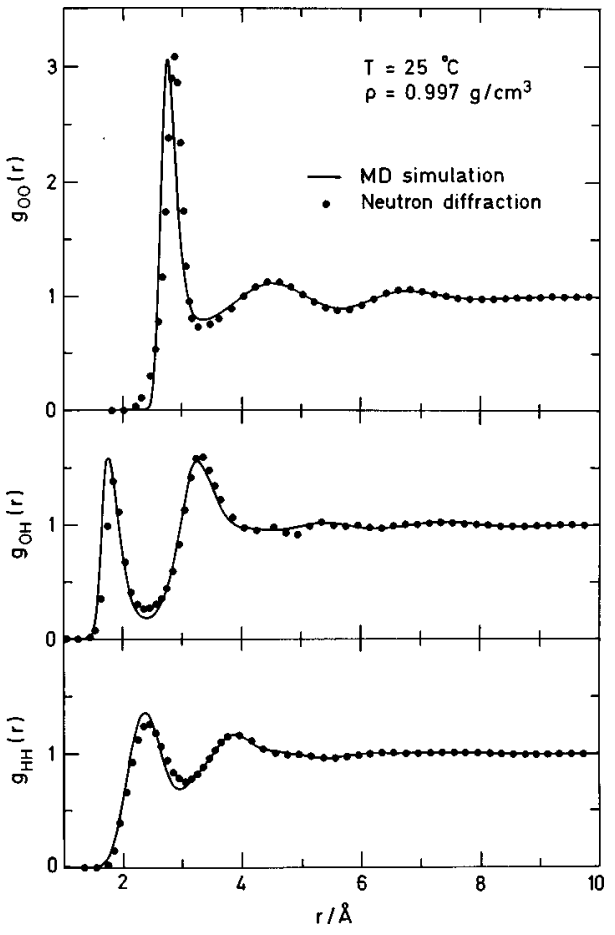

FIG. 2. Atom-atom distribution functions for water at ambient conditions from MD (SPCE model) and experiment (Ref. 22).

$$
U_{\mathrm{OO}}^{\mathrm{LJ}}(12)=-\left(\frac{A}{r}\right)^{6}+\left(\frac{B}{r}\right)^{12},
$$

with the parameters $A=3.7122 \AA(\mathrm{kJ} / \mathrm{mol})^{1 / 6}$ and $B=3.428$ $\AA(\mathrm{kJ} / \mathrm{mol})^{1 / 12}$. This gives a value of $\epsilon_{\mathrm{OO}} / k_{B}=78.197 \mathrm{~K}$ and $\sigma_{\mathrm{OO}}=3.1656 \AA$. In Fig. 2, we show the atom-atom distribution functions of water at room temperature from neutron scattering $^{22}$ and from a molecular dynamics (MD) simulation using the SPCE interaction. This comparison illustrates the ability of this simple interaction to model the structure of liquid water.

\section{RESULTS AND DISCUSSION}

All the integral equation calculations presented here have been carried out using 512 points with a grid size $\Delta r$ $=0.02 \sigma_{\mathrm{OO}}$ on the center-to-center distance $r$. In the spherical harmonic expansions, Eq. (4), we have used coefficients up to and including $X_{333}^{33}(r)$. All the simulations have been performed for a 256 molecule sample in the canonical ensemble ${ }^{15}$ using Berendsen's thermostat, ${ }^{23}$ with a time constant for temperature coupling of $0.4 \mathrm{ps}$ and a time step of 0.001 ps. A typical run comprises 10000 configurations for thermalization and 35000 for averaging of thermodynamic and structural properties. Given the success of the SPCE model in representing liquid water, we begin the study with ambient thermodynamic conditions, namely $\rho=0.997 \mathrm{~g} / \mathrm{cm}^{3}$ and $T=298.15 \mathrm{~K}$. For this state we use the full optimization procedure, Eq. (3), and derive atom-atom distribution functions from the coefficients of $g(12)$ using the procedure outlined in Ref. 14. The computed thermodynamic properties from this calculation are summarized in Table I, along with the corresponding MD data. The agreement is reasonable
TABLE I. Thermodynamic properties of the SPCE model of water at ambient conditions and above the critical temperature obtained from MD simulation and RHNC integral equation.

\begin{tabular}{ccccccc}
\hline \hline & & \multicolumn{2}{c}{$p / \mathrm{kbar}$} & & \multicolumn{2}{c}{$U /(\mathrm{kJ} / \mathrm{mol})$} \\
\cline { 3 - 4 } \cline { 6 - 7 }$T / \mathrm{K}$ & $\rho /\left(\mathrm{g} / \mathrm{cm}^{3}\right)$ & $\mathrm{MD}$ & $\mathrm{RHNC}$ & & $\mathrm{MD}$ & $\mathrm{RHNC}$ \\
\hline 298.15 & 0.997 & $0.00(0.54)$ & -0.06 & & $-46.79(0.21)$ & -42.06 \\
700.00 & 0.320 & $0.32(0.21)$ & 0.36 & & $-17.63(0.54)$ & -17.71 \\
700.00 & 1.000 & $7.86(0.98)$ & 8.88 & & $-32.57(0.44)$ & -30.50 \\
\hline \hline
\end{tabular}

although not remarkable. The calculated structure, on the other hand, (seen in Fig. 3), is decidedly poor, particularly for the $\mathrm{O}-\mathrm{O}$ distribution function. Moreover, comparison with results from the ordinary hypernetted chain (HNC) approximation, with $B_{0}(12)=0$, suggests that the reference hard-sphere bridge function has little effect on the structure. In the $\mathrm{O}-\mathrm{H}$ distribution function, we see that the position of the $\mathrm{OH}$ hydrogen bond is correctly located. As with $g_{\mathrm{HH}}(r)$, however, the amplitudes are off.

The notable mismatch of the $\mathrm{O}-\mathrm{O}$ structure needs some explanation. To gain insight into the temperature dependence of the pair structure of water, we have performed several MD runs at $\rho=1 \mathrm{~g} / \mathrm{cm}^{3}$ and $T$ ranging from 300 to $700 \mathrm{~K}$. The $g_{\text {oo }}(r)$ curves from these calculations are displayed in Fig. 4. They exhibit a rather peculiar behavior, in that the $700 \mathrm{~K}$ distribution function seems to have somewhat more structure than the $400 \mathrm{~K}$ curve, while an abrupt change occurs at 300 $\mathrm{K}$ where considerably more pair structure shows up. The integral equation results for $g_{\mathrm{OO}}(r)$ in Fig. 3 appear to fit those of $\mathrm{MD}$ at a higher temperature. Actually, when one compares the RHNC results for $700 \mathrm{~K}$ with those of simulation at the same temperature, the agreement is seen to be

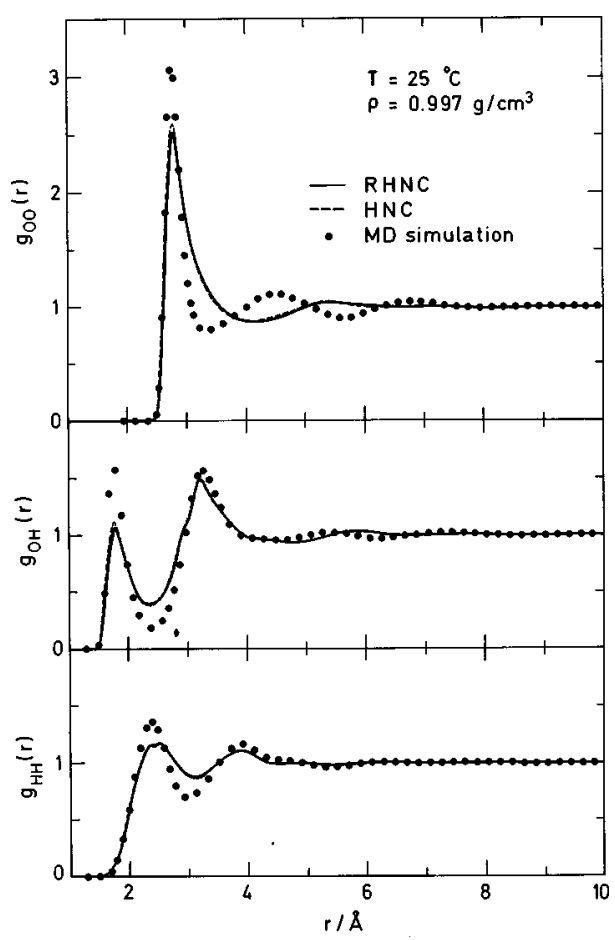

FIG. 3. Atom-atom distribution functions for SPCE water at ambient conditions from MD (solid circles), RHNC (solid lines), and HNC (dashed lines). 


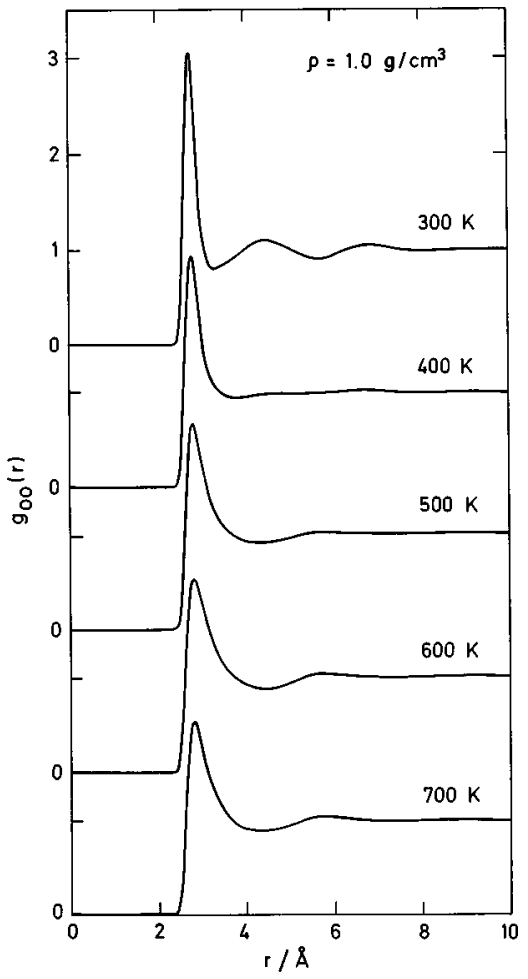

FIG. 4. MD data for the oxygen-oxygen distribution function in SPCE water along the $\rho=1 \mathrm{~g} / \mathrm{cm}^{3}$ isochore for various temperatures.

excellent (Fig. 5). If we now move to the supercritical region shown in Fig. $6\left(\rho=0.32 \mathrm{~g} / \mathrm{cm}^{3}, T=700 \mathrm{~K}\right)$, we find the integral equation continues to provide excellent estimates. This agreement applies to the thermodynamic properties as well (see Table I). Now, keeping $\rho$ fixed at $0.32 \mathrm{~g} / \mathrm{cm}^{3}$, we

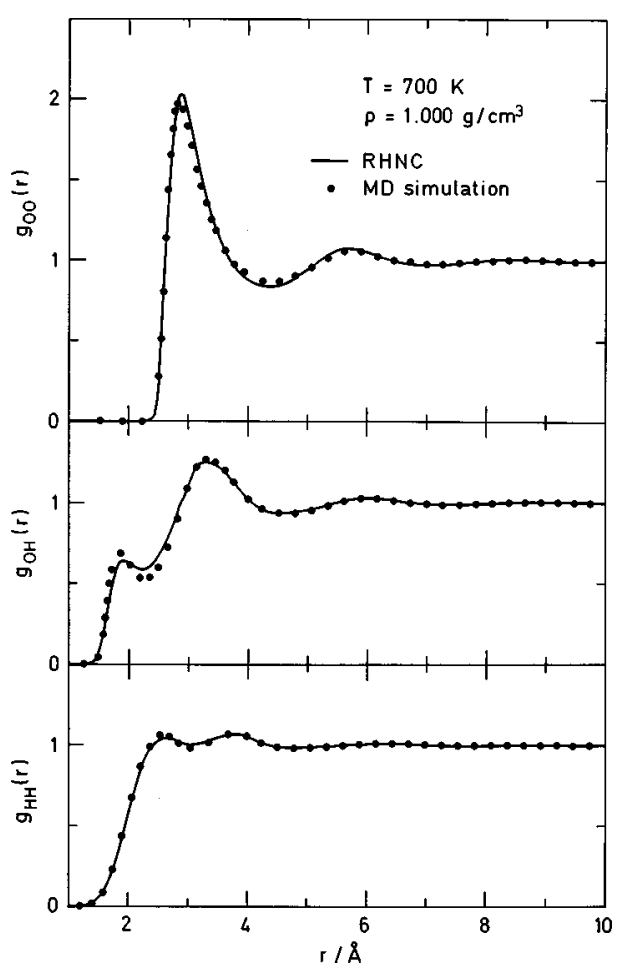

FIG. 5. Atom-atom distribution functions for high temperature $(700 \mathrm{~K})$ SPCE water from MD (solid circles) and RHNC (solid lines).

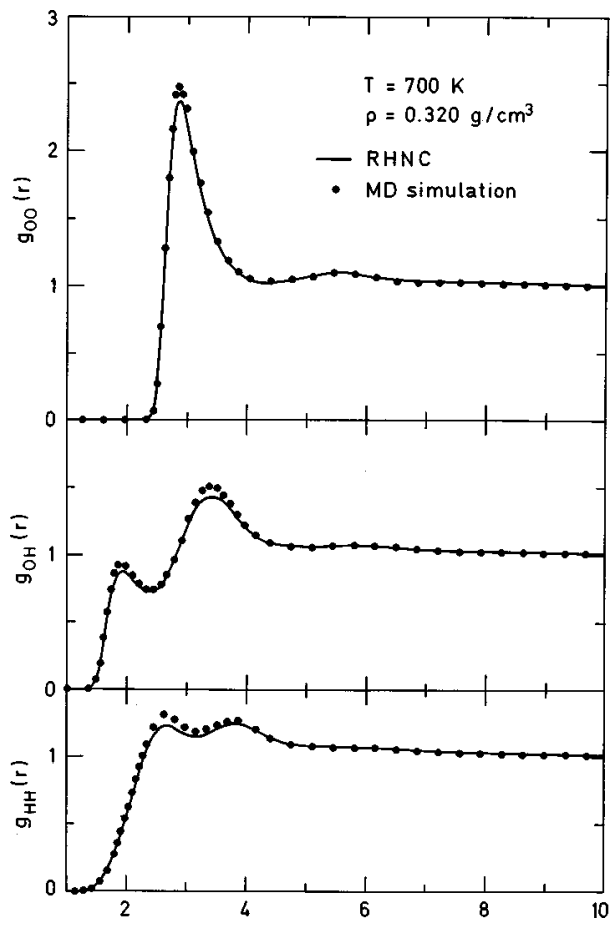

FIG. 6. Atom-atom distribution functions for supercritical SPCE water from MD (solid circles) and RHNC (solid lines).

proceed to lower the temperature and monitor the behavior of the isothermal compressibility. The evolution with temperature of this quantity can be observed in Fig. 7. We see that the estimate for the critical temperature $T_{c}=678 \mathrm{~K}$ is not far off from the Gibbs ensemble Monte Carlo results of Guissani and Guillot, ${ }^{9} T_{c}^{\mathrm{GEMC}}=657 \mathrm{~K}$. For the critical pressure we obtain $p_{c}=303$ bar, which is of the same order as the proposed estimates ${ }^{9} p_{c}^{\mathrm{MC}}=160-189$ bar. Further, we obtain $\varepsilon=5.6$ for the dielectric constant at the critical point, in good agreement with the value reported by Guissani and Guillot, ${ }^{9}$ $\varepsilon^{\mathrm{MC}}=6$. This last datum stands in contrast with the poor result furnished by the theory at ambient conditions, $\varepsilon$ $=53$, well below the simulation value of $\varepsilon^{\mathrm{MC}}=81$. Taking

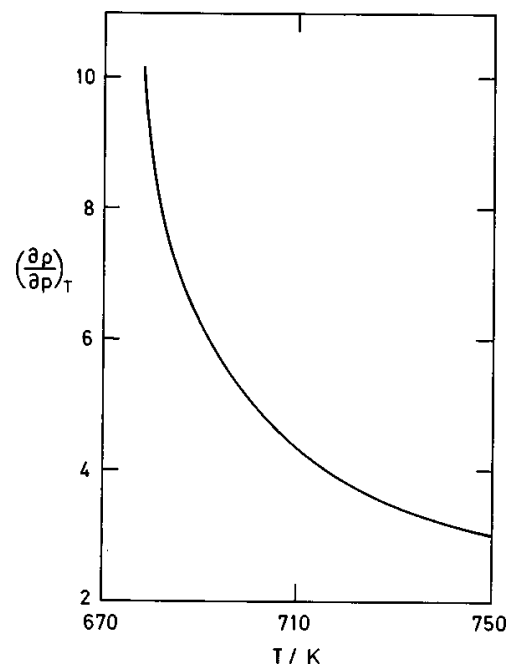

FIG. 7. Temperature variation of the isothermal compressibility near the critical point at density $\rho=0.32 \mathrm{~g} / \mathrm{cm}^{3}$ in RHNC approximation. 


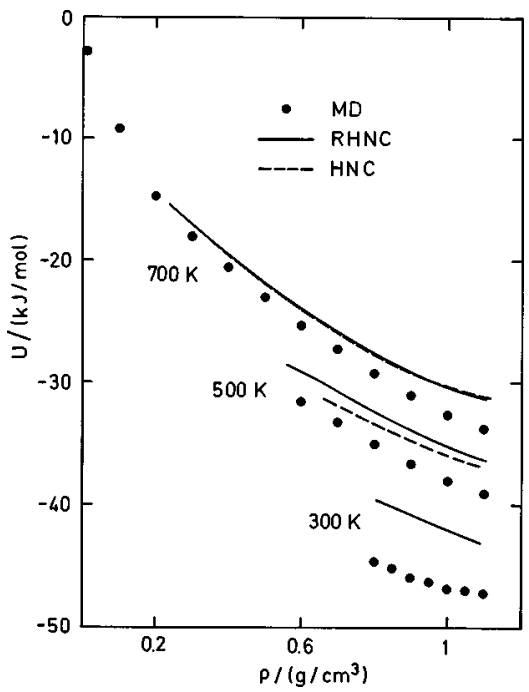

FIG. 8. Internal energy for SPCE water along various isotherms from MD (solid circles), RHNC (solid lines), and HNC (dashed lines).

into account the deviations exhibited by the theoretical distribution functions seen in Fig. 3, it would be surprising if a quantity as closely linked with the orientational order of the fluid as is the dielectric constant would be reproduced with better accuracy.

In order to assess the overall behavior of the integral equation results, we have performed calculations along several isotherms. The resulting internal energies and pressures are plotted in Figs. 8 and 9, respectively. For comparison, we also include results from the HNC equation. It turns out that the HNC approximation seems to have no physical solution for $T=300 \mathrm{~K}$. For the RHNC approximation at $300 \mathrm{~K}$, it is striking that the pressures are rather accurate while energy values show discrepancies of about $10 \%$. But what is more surprising is that on average the approximation should be so much better for the thermodynamics overall than for the structure at low temperature. For the sake of completeness, we include in Fig. 10 the zero pressure curve comparing

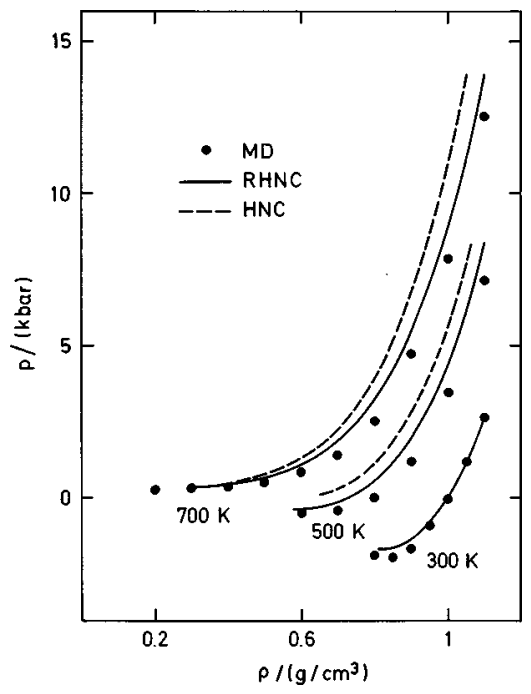

FIG. 9. Pressure for SPCE water along various isotherms from MD (solid circles), RHNC (solid lines), and HNC (dashed lines).

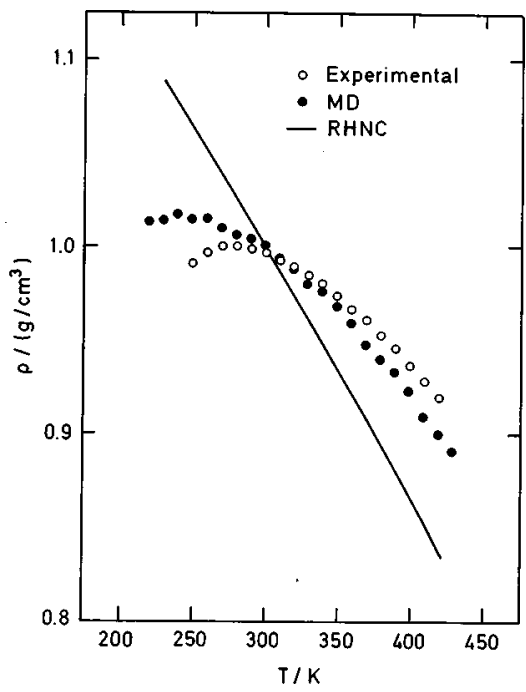

FIG. 10. The density maximum in water at zero pressure. Empty circles correspond to experimental data from Ref. 24, filled circles to NPT MD calculations, and the solid line to RHNC results.

results from theory, $\mathrm{MD}$ in the isothermal-isobaric ensemble, ${ }^{15}$ and experiment. ${ }^{24}$ For the MD runs, we have also used Berendsen's thermostat and barostat, ${ }^{23}$ with time constants of $0.4 \mathrm{ps}$ for temperature coupling and $0.5 \mathrm{ps}$ for pressure coupling. Regarding the integral equation, the zero pressure results are obtained by fine tuning the density until the virial pressure vanishes. These are perhaps the thermodynamic conditions where discrepancies are most apparent. The theoretical calculation does not exhibit at all the very significant density maximum, which is found both experimentally and in the simulation.

To find an answer to these puzzles one must go beyond the pair structure. Thus, we have extracted from the simulations the three-body distribution function $g_{\mathrm{OOO}}^{(3)}\left(r_{12}, r_{23}, \theta_{123}\right)$ for $r_{12}=r_{23}=2.75 \AA$, which corresponds to the position of the maximum in the pair function $g_{\mathrm{OO}}(r)$. We plot this quantity in Fig. 11 as a function of $\theta_{123}$, the

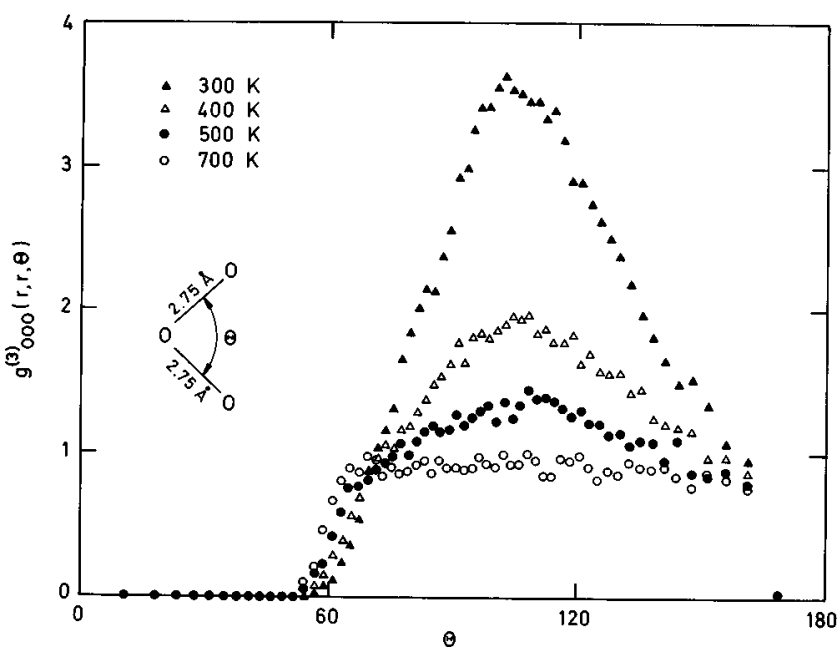

FIG. 11. Three-body distribution function between oxygen atoms from MD simulation for $r_{12}=r_{13}=2.75 \AA$, which corresponds to the maximum in $g_{\mathrm{OO}}(r)$. 
angleformed by the vectors $\mathbf{r}_{12}$ and $\mathbf{r}_{23}$ (vectors that in this case go from $\mathrm{O}$ atom to $\mathrm{O}$ atom); here we see the evolution in this distribution with temperature. The growth of the maximum from $\theta_{\mathrm{OOO}}=100^{\circ}$ to $120^{\circ}$ indicates the buildup of tetrahedral ordering. This tetrahedral ordering is averaged out in the pair functions and that is why $g_{\mathrm{OO}}(r)$ at $400 \mathrm{~K}$ is less structured than $g_{\mathrm{OO}}(r)$ at $700 \mathrm{~K}$ (see Fig. 4). The absence of the second neighbor peaks is characteristic of fluids with tetrahedral local order, as in the case of liquid and amorphous silicon. ${ }^{25}$ This appears to be the core of the problem. At room temperature SPCE water is close to the freezing point and the particles tend to organize in open structures that locally present the ordering characteristics of the solid. From the work of Rosenfeld, ${ }^{26}$ we know that the reference system bridge function should contain the basic geometric relations of the system, and hence it is obvious that the hard sphere fluid, which crystallizes in closed packed structures, is a poor candidate to account for the geometric features of loosely coordinated systems like water at room temperature. Although it is the pair potential that induces the tetrahedral ordering, of all the pair functions involved in the closure relation [Eq. (2)] only the bridge function directly accounts for the effect of $N$-body correlations $(N \geqslant 3)$. Also, since the density maximum in water is closely connected with changes in the local coordination numbers, it is easy to understand the inability of the spherically symmetric bridge function to capture these crucial features. On the other hand, in other systems with strong local order like acetonitrile ${ }^{27}$ the HNC seems to be reliable even at low temperatures where the ordering starts to resemble that of the solid. The same can be said about the performance of the HNC for acetone and chloroform ${ }^{20}$ which exhibit quite large spatial correlations. However, none of these systems crystallizes in tetrahedral arrangements, and in all their center-to-center pair distribution functions the second neighbor peak is clearly visible. It is obvious that further investigations are needed to thoroughly understand how local geometries influence the performance of integral equation approximations.

In summary, we have investigated the behavior of the molecular RHNC theory with hard-sphere bridge function for the SPCE model of water and find that, while the theory performs fairly well in the moderate to high temperature regime both for the structure and thermodynamics, the computed microscopic structure - in particular $g_{\mathrm{OO}}(r)$ - at room temperature shows large discrepancies. An analysis of the oxygen three-body function, $g_{\mathrm{OOO}}^{(3)}\left(r_{12}, r_{23}, \theta_{123}\right)$, indicates that these discrepancies might well be ascribed to the buildup of tetrahedral ordering, which cannot be properly incorporated into the RHNC approximation via a spherically symmetric hard-sphere bridge function. Future work should thus aim at incorporating those geometric features into the reference bridge function.

\section{ACKNOWLEDGMENTS}

This work was supported in part by the Dirección General de Enseñanza Superior, Spain under Grant No. PB940112. F. L. thanks the Dirección General de Enseñanza Superior, Spain for sabbatical support under Grant No. SAB950373 during the early part of this work.

${ }^{1}$ K. Laasonen, M. Sprik, M. Parrinello, and R. Car, J. Chem. Phys. 99, 9080 (1993).

${ }^{2}$ W. L. Jorgensen, J. Chem. Phys. 77, 4156 (1982).

${ }^{3}$ H. J. C. Berendsen, J. P. M. Postma, W. F. van Gunsteren, and J. Hermans, in Intermolecular Forces, edited by B. Pullmann (Reidel, Dordrecht, 1981).

${ }^{4}$ H. J. C. Berendsen, J. R. Grigera, and T. P. Straatsma, J. Phys. Chem. 91, 6269 (1987).

${ }^{5}$ G. C. Lie and E. Clementi, Phys. Rev. A 33, 2679 (1986).

${ }^{6}$ J. W. Halley, J. R. Rustad, and A. Rahman, J. Chem. Phys. 98, 4110 (1993); I. M. Svishchev, P. G. Kusalik, J. Wang, and R. J. Boud, ibid. 105, 4742 (1996); A. A. Chialvo and P. T. Cummings, ibid. 105, 8274 (1996); L. M. Dang and T. Chang, ibid. 106, 8149 (1997).

${ }^{7}$ H. L. Lemberg and F. H. Stillinger, J. Chem. Phys. 62, 1677 (1975).

${ }^{8}$ B. Guillot, J. Chem. Phys. 95, 1543 (1991), and references therein.

${ }^{9}$ Y. Guissani and B. Guillot, J. Chem. Phys. 98, 8221 (1993).

${ }^{10}$ B. M. Pettitt and P. J. Rossky, J. Chem. Phys. 77, 1451 (1982).

${ }^{11}$ D. Chandler, R. Silbey, and B. M. Ladanyi, Mol. Phys. 46, 1335 (1982).

${ }^{12}$ L. Lue and D. Blankschtein, J. Chem. Phys. 102, 5427 (1995).

${ }^{13}$ F. Lado, E. Lomba, and M. Lombardero, J. Chem. Phys. 103, 481 (1995).

${ }^{14}$ M. Alvarez, F. Lado, E. Lomba, M. Lombardero, and C. Martín, J. Chem. Phys. 107, 4642 (1997).

${ }^{15}$ All MD results presented in this paper have been generated using the DL_POLY program. DL_POLY is a package of molecular simulation routines written by W. Smith and T. R. Forester. Copyright: The Engineering and Physical Sciences Research Council, acting through its Daresbury and Rutherford Appleton Laboratory at Daresbury Laboratory, 1994.

${ }^{16}$ F. Lado, Phys. Lett. A 89, 196 (1982); J. Chem. Phys. 81, 4592 (1984).

${ }^{17}$ L. Verlet and J. J. Weis, Phys. Rev. A 5, 939 (1972).

${ }^{18}$ D. Henderson and E. W. Grundke, J. Chem. Phys. 63, 601 (1975).

${ }^{19}$ M. E. Rose, Elementary Theory of Angular Momentum (Wiley, New York, 1957)

${ }^{20}$ J. Richardi, P. H. Fries, R. Fischer, S. Rast, and H. Krienke, Mol. Phys. 93, 925 (1998).

${ }^{21}$ M. Lombardero, C. Martín, and E. Lomba, J. Chem. Phys. 97, 2724 (1992).

${ }^{22}$ A. K. Soper and M. G. Phillips, Chem. Phys. 107, 47 (1986).

${ }^{23}$ H. J. C. Berendsen, J. P. M. Postma, W. F. van Gunsteren, A. DiNola, and J. Haak, J. Chem. Phys. 81, 3684 (1984).

${ }^{24}$ G. S. Kell, J. Chem. Eng. Data 20, 97 (1975).

${ }^{25}$ F. H. Stillinger and T. A. Weber, Phys. Rev. B 31, 5262 (1985).

${ }^{26}$ Y. Rosenfeld, Phys. Rev. Lett. 72, 3831 (1994).

${ }^{27}$ P. H. Fries, W. Kunz, P. Calmettes, and P. Turq, J. Chem. Phys. 101, 554 (1994); P. H. Fries, J. Richardi, R. Fischer, S. Rast, and H. Krienke, J. Mol. Liq. 73\&74, 465 (1997). 